Bull. Korean Math. Soc. 48 (2011), No. 2, pp. 397-412

DOI 10.4134/BKMS.2011.48.2.397

\title{
SPATIAL BEHAVIOR OF SOLUTION FOR THE STOKES FLOW EQUATION
}

\author{
Yan Liu, Wenhui Liao, and Changhao Lin
}

\begin{abstract}
In this paper, the equation of the transient Stokes flow of an incompressible viscous fluid is studied. Growth and decay estimates are established associating some appropriate cross sectional line and area integral measures. The method of the proof is based on a first-order differential inequality leading to an alternative of Phragmén-Lindelöf type in terms of an area measure of the amplitude in question. In the case of decay, we also indicate how to bound the total energy.
\end{abstract}

\section{Introduction}

In 1960-1980's, principle of Saint-Venant type used to be one of the most popular subjects of applied mathematics and mechanics. A great number of investigation results have expanded enormously the classical Saint-Venant principle. A review of recent work on Saint-Venant's principle is given in the work of Horgan and Knowles [6], and has been periodically updated by Horgan [3], [4]. A common feature of Saint-Venant type theorems is to establish the exponential decay estimates of energy with axial distance from the near end of a semi-infinite strip or cylinder.

Knowles [9] established exponential decay estimates for solution of the biharmonic equation in his study of Saint-Venant's principle in plane isotropic elastostatics for bounded, simply connected domain of general shape. Since then, many authors have investigated the same problems in a semi-infinite strip (see [5], [3] and the references cited therein). Their common goal has been to try to establish an energy decay results. It is well known that another interprelation for the biharmonic equation in the plane is that of the stream function

Received September 7, 2009.

2010 Mathematics Subject Classification. 35B40, 35K10, 35K20.

Key words and phrases. spatial behavior, transient Stokes flow, Saint-Venant's principle, biharmonic equation, stream function.

The work was supported by the national natural Science Foundation of China (Grants 10971234, 11001088, 11026227), Foundation for Distinguished Young Talents in Higher Education of Guangdong, China (Grant LYM10100), and Science Foundation of Guangdong University of Finance (Grant 10XJ03-07). 
in two dimensional Stokes flow, hence the results of the Saint-Venant's principle in plane elastostatics are also relevant to the study of the spatial evolution of stationary stokes flows in a semi-infinite paralled plate channel. Numerous authors have dealt with Saint-Venant type decay estimate for solutions of the biharmonic equations in a semi-infinite channel in $\mathbb{R}^{2}$, we mention in particular the papers of Flavin [1], Horgan [5], Knowles [8] and Flavin et al. [2]. In a paper [10], Lin has established energy decay estimates for solutions of the plane Stokes flow in a semi-infinite channel, subject to nonzero boundary conditions on the end only. Such estimates yielding an exponential decay of energy with axial distance from the end are based on differential inequality techniques developed by Knowles [9], and Horgan and Knowles [6] in their investigations of Saint-Venant's principle in classical elasticity theory. Recently, Song [13] investigated the same time-dependent Stokes flow problem and obtained an analogous result with an improved decay rate. The papers concerning with biharmonic equations may be viewed as a version of Saint-Venant's principle in steady state or transient stokes flow, but common to all was the assumption that the solution must satisfy some a priori decay criterion at infinity. One would like to remove this a priori restriction. One way to do this is to derive an appropriate Phragmén-Lindelöf alternative.

The classical Phragmén-Lindelöf theorem states that harmonic function which vanish on the cylindrical surface must either grow exponential or decay exponential with distance from the finite end of the cylinder. Phragmén-Lindelöf type alternative results for harmonic functions were obtained by Horgan et al. [7] under non-linear boundary conditions on the lateral surface of a semi-infinite cylinder. Particularly, Payne et al. [12] established the Phragmén-Lindelöf type results in three type special domains in $\mathbb{R}^{2}$. Additional references for Phragmén-Lindelöf type results may be found in [11] and [4] therein.

In this paper, we investigate the spatial behaviour for the equation of the transient Stokes flow of an incompressible viscous fluid. We use a stream function to transform the equation to a biharmonic equation, unlike many other papers deal with the biharmonic equation, our method of the proof is based on a first-order differential inequality not the second-order differential inequality. In the next section we will formulate the initial and boundary value problem that serves as the basis for our analysis. Section 3 is devoted to deriving the Phragmén-Lindelöf type alternative results. In the final section we will indicate how to bound the total energy.

In the present paper, the comma is used to indicate partial differentiation and the differentiation with respect to the direction $x_{k}$ is denoted as, $k$, thus $u_{, \alpha}$ denotes $\frac{\partial u}{\partial x_{\alpha}}$, and $u_{, t}$ denotes $\frac{\partial u}{\partial t}$. The usual summation convection is employed with repeated Greek subscripts $\alpha$ summed from 1 to 2 . Hence, $u_{\alpha, \alpha}=$ $\sum_{\alpha=1}^{2} \frac{\partial u_{\alpha}}{\partial x_{\alpha}}$. 


\section{Basic equation}

We consider the time-dependent Stokes equations governing the transient slow flow of an incompressible slow viscous fluid on an unbounded region $\Omega_{0}$ defined by

$$
\Omega_{0}:=\left\{\left(x_{1}, x_{2}\right) \mid x_{1}>0,0<x_{2}<h\right\},
$$

where $h$ is a fixed constant, and we introduce the notation

$$
L_{z}=\left\{\left(x_{1}, x_{2}\right) \mid x_{1}=z \geq 0,0 \leq x_{2} \leq h\right\} .
$$

The velocity field $v_{\alpha}\left(x_{1}, x_{2}, t\right)$ and the pressure $p\left(x_{1}, x_{2}, t\right)(\alpha=1,2)$ for the transient Stokes flow of an incompressible viscous fluid are to be classical solutions of the initial-boundary value problem:

$$
\begin{gathered}
v_{\alpha, t}=\nu \Delta v_{\alpha}-p_{, \alpha} \quad \text { in } \Omega_{0} \times[0, \infty), \\
v_{\alpha, \alpha}=0 \quad \text { in } \quad \Omega_{0} \times[0, \infty), \\
v_{\alpha}\left(x_{1}, 0, t\right)=v_{\alpha}\left(x_{1}, h, t\right)=0 \quad \alpha=1,2, \\
v_{\alpha}\left(0, x_{2}, t\right)=f_{\alpha}\left(x_{2}, t\right) \quad \alpha=1,2 . \\
v_{\alpha}\left(x_{1}, x_{2}, 0\right)=0 \quad\left(x_{1}, x_{2}\right) \in \Omega_{0},
\end{gathered}
$$

where $\Delta$ is the two dimensional Laplace operator and $\nu$ is the constant kinematic viscosity. The functions $f_{\alpha}\left(x_{2}, t\right)$ are assumed to satisfy the compatibility $f_{\alpha}(0, t)=f_{\alpha}(h, t)=0$. For simplicity, it is assumed that $\int_{L_{0}} f_{1} d x_{2}=0$ for all $t>0$.

In order to eliminate the pressure term $p_{, \alpha}$, we introduce the stream function $u\left(x_{1}, x_{2}, t\right)$ such that

$$
v_{1}=u_{, 2}, v_{2}=-u_{, 1} .
$$

So the equations (2.2)-(2.6) are transformed into the following fourth order initial-boundary value problem:

$$
\begin{gathered}
\Delta^{2} u=(\Delta u)_{, t} \quad \text { in } \quad \Omega_{0}, \\
u\left(x_{1}, 0, t\right)=u_{n}\left(x_{1}, 0, t\right)=0 \quad x_{1}>0, t>0, \\
u\left(x_{1}, h, t\right)=u_{n}\left(x_{1}, h, t\right)=0 \quad x_{1}>0, t>0, \\
u\left(0, x_{2}, t\right)=g_{1}\left(x_{2}, t\right)=\int_{0}^{x_{2}} f_{1}(s, t) d s \quad 0 \leq x_{2} \leq h, t>0, \\
u_{, 1}\left(0, x_{2}, t\right)=g_{2}\left(x_{2}, t\right)=-f_{2}\left(x_{2}, t\right) \quad 0 \leq x_{2} \leq h, t>0, \\
u_{, \alpha}\left(x_{1}, x_{2}, 0\right)=0 \quad x_{1}>0,0<x_{2}<h,
\end{gathered}
$$

where we make no assumption on $u$ as $x_{1} \rightarrow \infty$. Here $\Delta$ is the harmonic operator, and $\Delta^{2}$ is the biharmonic operator, $u_{n}$ is the outward normal derivative, 
and we adapt the standard notations, i.e., $u_{, i}=\frac{\vartheta u}{\vartheta x_{i}}, u_{, t}=\frac{\vartheta u}{\vartheta t}$. The differentiable functions $g_{1}$ and $g_{2}$ are prescribed and assumed to satisfy appropriate compatibility conditions: $g_{1}(0, t)=g_{1}(h, t)=g_{2}(0, t)=g_{2}(h, t)=g_{1}^{\prime}(0, t)=$ $g_{1}^{\prime}(h, t)=0$, where $g_{1}^{\prime}$ denotes the partial differentiation with respect to $x_{2}$.

We define energy expressions of the forms:

$$
E_{1}(z, t)=2 \int_{0}^{t} \int_{z_{1}}^{z} \int_{L_{\xi}} u_{, \alpha \eta} u_{, \alpha \eta} d A d \eta+\left.\int_{z_{1}}^{z} \int_{L_{\xi}} u_{, \alpha \beta} u_{, \alpha \beta} d A\right|_{\eta=t}
$$

$$
E_{2}(z, t)=\left.\frac{1}{2} \int_{0}^{z} \int_{L_{\xi}} u_{, \alpha \beta} u_{, \alpha \beta} d A\right|_{\eta=t}+\left.\frac{1}{2} \int_{0}^{z} \int_{L_{\xi}} u_{, 1 \alpha} u_{, 1 \alpha} d A\right|_{\eta=t},
$$

where we use the summation convention on repeated indices and the Greek subscripts range over 1,2 . Our purpose is to determine the alternative that either $E_{1}(z, t)$ grows exponentially or $E_{2}(z, t)$ decays exponentially as $z \rightarrow \infty$.

We start our analysis by the following identity. On using the divergence theorem and the initial-boundary conditions (2.10)-(2.14), it leads to

$$
\begin{aligned}
0= & \int_{0}^{t} \int_{0}^{z} \int_{L_{\xi}} u_{, \eta}\left(\Delta u_{, \eta}-\Delta^{2} u\right) d A d \eta \\
= & -\int_{0}^{t} \int_{0}^{z} \int_{L_{\xi}} u_{, \alpha \eta} u_{, \alpha \eta} d A d \eta+\int_{0}^{t} \int_{L_{z}} u_{, \eta} u_{, 1 \eta} d x_{2} d \eta \\
& -\int_{0}^{t} \int_{L_{0}} u_{, \eta} u_{, 1 \eta} d x_{2} d \eta+\int_{0}^{t} \int_{0}^{z} \int_{L_{\xi}} u_{, \alpha \eta} u_{, \alpha \beta \beta} d A d \eta \\
& -\int_{0}^{t} \int_{L_{z}} u_{, \eta} u_{, 1 \beta \beta} d x_{2} d \eta+\int_{0}^{t} \int_{L_{0}}^{u_{, \eta} u_{, 1 \beta \beta} d x_{2} d \eta} \\
= & -\int_{0}^{t} \int_{0}^{z} \int_{L_{\xi}} u_{, \alpha \eta} u_{, \alpha \eta} d A d \eta+\int_{0}^{t} \int_{L_{z}} u_{, \eta} u_{, 1 \eta} d x_{2} d \eta \\
& -\int_{0}^{t} \int_{L_{0}}^{z} u_{, \eta} u_{, 1 \eta} d x_{2} d \eta-\int_{0}^{t} \int_{0}^{\int_{L_{\xi}}} u_{, \alpha \beta \eta} u_{, \alpha \beta} d A d \eta \\
& +\int_{0}^{t} \int_{L_{z}} u_{, \alpha \eta} u_{, 1 \alpha} d x_{2} d \eta-\int_{0}^{t} \int_{L_{0}}^{u_{, \alpha \eta} u_{, 1 \alpha} d x_{2} d \eta} \\
& -\int_{0}^{t} \int_{L_{z}} u_{, \eta} u_{, 1 \beta \beta} d x_{2} d \eta+\int_{0}^{t} \int_{L_{0}}^{u_{, \eta} u_{, 1 \beta \beta} d x_{2} d \eta .}
\end{aligned}
$$

From (2.17), we define

$$
\phi_{1}(z, t)=-\int_{0}^{t} \int_{L_{z}}\left(u_{, \eta} u_{, 1 \eta}+u_{, \alpha \eta} u_{, 1 \alpha}-u_{, \eta} u_{, 1 \beta \beta}\right) d x_{2} d \eta
$$


SPATIAL BEHAVIOR OF SOLUTION FOR THE STOKES FLOW EQUATION 401

(2.18) $=-\int_{0}^{t} \int_{0}^{z} \int_{L_{\xi}} u_{, \alpha \eta} u_{, \alpha \eta} d A d \eta-\left.\frac{1}{2} \int_{0}^{z} \int_{L_{\xi}} u_{, \alpha \beta} u_{, \alpha \beta} d A\right|_{\eta=t}+\phi_{1}(0, t)$.

Deriving as (2.17), we obtain

$$
\begin{aligned}
0= & \int_{0}^{t} \int_{0}^{z} \int_{L_{\xi}} u_{, 11}\left(\Delta u_{, \eta}-\Delta^{2} u\right) d A d \eta \\
= & -\int_{0}^{t} \int_{0}^{z} \int_{L_{\xi}} u_{, 1 \alpha \beta} u_{, 1 \alpha \beta} d A d \eta-\left.\frac{1}{2} \int_{0}^{z} \int_{L_{\xi}} u_{, 1 \alpha} u_{, 1 \alpha} d A\right|_{\eta=t} \\
& +\int_{0}^{t} \int_{L_{z}}\left(u_{, \alpha \alpha} u_{, \alpha \alpha 1}+u_{, 12} u_{, 2 \eta}\right) d x_{2} d \eta \\
& -\int_{0}^{t} \int_{L_{0}}\left(u_{, \alpha \alpha} u_{, \alpha \alpha 1}+u_{, 12} u_{, 2 \eta}\right) d x_{2} d \eta
\end{aligned}
$$

From (2.19), we define

$$
\begin{aligned}
\phi_{2}(z, t) & =-\int_{0}^{t} \int_{L_{z}}\left(u_{, \alpha \alpha} u_{, 1 \alpha \alpha}+u_{, 12} u_{, 2 \eta}\right) d x_{2} d \eta \\
& =-\int_{0}^{t} \int_{0}^{z} \int_{L_{\xi}} u_{, 1 \alpha \beta} u_{, 1 \alpha \beta} d A d \eta-\left.\frac{1}{2} \int_{0}^{z} \int_{L_{\xi}} u_{, 1 \alpha} u_{, 1 \alpha} d A\right|_{\eta=t}+\phi_{2}(0, t) .
\end{aligned}
$$

We now introduce the function

$$
\begin{aligned}
\phi(z, t)= & \phi_{1}(z, t)+\phi_{2}(z, t) \\
= & -\int_{0}^{t} \int_{L_{z}}\left(u_{, \eta} u_{, 1 \eta}+u_{, \alpha \eta} u_{, 1 \alpha}-u_{, \eta} u_{, 1 \beta \beta}\right) d x_{2} d \eta \\
& -\int_{0}^{t} \int_{L_{z}}\left(u_{, \alpha \alpha} u_{, 1 \alpha \alpha}+u_{, 12} u_{, 2 \eta}\right) d x_{2} d \eta \\
= & -\int_{0}^{t} \int_{0}^{z} \int_{L_{\xi}} u_{, \alpha \eta} u_{, \alpha \eta} d A d \eta-\left.\frac{1}{2} \int_{0}^{z} \int_{L_{\xi}} u_{, \alpha \beta} u_{, \alpha \beta} d A\right|_{\eta=t}+\phi_{1}(0, t) \\
& -\int_{0}^{t} \int_{0}^{z} \int_{L_{\xi}} u_{, 1 \alpha \beta} u_{, 1 \alpha \beta} d A d \eta-\left.\frac{1}{2} \int_{0}^{z} \int_{L_{\xi}} u_{, 1 \alpha} u_{, 1 \alpha} d A\right|_{\eta=t}+\phi_{2}(0, t) .
\end{aligned}
$$

Obviously, from the definition of $\phi(z, t)$, we obtain

$$
\frac{\partial}{\partial z} \phi(z, t) \leq 0
$$

From the definition of (2.21), we also have for arbitrary $z>z_{0} \geq 0$,

$$
\phi(z, t)-\phi\left(z_{0}, t\right)=-\int_{0}^{t} \int_{z_{0}}^{z} \int_{L_{\xi}} u_{, \alpha \eta} u_{, \alpha \eta} d A d \eta-\left.\frac{1}{2} \int_{z_{0}}^{z} \int_{L_{\xi}} u_{, \alpha \beta} u_{, \alpha \beta} d A\right|_{\eta=t}
$$




$$
-\int_{0}^{t} \int_{z_{0}}^{z} \int_{L_{\xi}} u_{, 1 \alpha \beta} u_{, 1 \alpha \beta} d A d \eta-\left.\frac{1}{2} \int_{z_{0}}^{z} \int_{L_{\xi}} u_{, 1 \alpha} u_{, 1 \alpha} d A\right|_{\eta=t} .
$$

From (2.23), we can see that if $\phi(z, t) \longrightarrow 0$ as $z \longrightarrow \infty$, then

$$
\begin{aligned}
\phi(z, t)= & \int_{0}^{t} \int_{z}^{\infty} \int_{L_{\xi}} u_{, \alpha \eta} u_{, \alpha \eta} d A d \eta+\left.\frac{1}{2} \int_{z}^{\infty} \int_{L_{\xi}} u_{, \alpha \beta} u_{, \alpha \beta} d A\right|_{\eta=t} \\
& +\int_{0}^{t} \int_{z}^{\infty} \int_{L_{\xi}} u_{, 1 \alpha \beta} u_{, 1 \alpha \beta} d A d \eta+\left.\frac{1}{2} \int_{z}^{\infty} \int_{L_{\xi}} u_{, 1 \alpha} u_{, 1 \alpha} d A\right|_{\eta=t} .
\end{aligned}
$$

On the other hand, if $-\phi(z, t)$ is unbounded below by a positive number $\chi(z, t)$, then

$$
\begin{aligned}
& \int_{0}^{t} \int_{0}^{z} \int_{L_{\xi}} u_{, \alpha \eta} u_{, \alpha \eta} d A d \eta+\left.\frac{1}{2} \int_{0}^{z} \int_{L_{\xi}} u_{, \alpha \beta} u_{, \alpha \beta} d A\right|_{\eta=t} \\
& +\int_{0}^{t} \int_{0}^{z} \int_{L_{\xi}} u_{, 1 \alpha \beta} u_{, 1 \alpha \beta} d A d \eta+\left.\frac{1}{2} \int_{0}^{z} \int_{L_{\xi}} u_{, 1 \alpha} u_{, 1 \alpha} d A\right|_{\eta=t} \\
\geq & \chi(z, t)+\phi(0, t) .
\end{aligned}
$$

In the following part, we will use the following inequality: Clearly, we know, for $u(z, 0, t)=0$, we have

$$
u\left(z, x_{2}, t\right)=\int_{0}^{x_{2}} u_{, s}(z, s, t) d s,
$$

thus, using Schwarz's inequality, we easily find that

$$
\int_{L_{z}} u^{2} d x_{2} \leq h^{2} \int_{L_{z}} u_{, 2}^{2} d x_{2} .
$$

\section{Phragmén-Lindelöf type alternative results}

In this part, we'll use the technique which was used by Lin and Payne in [11]. For $t>0$, let $\tilde{t}$ be a value of time between 0 and $t$, at which function

$$
m(z, t)=\left.\frac{1}{2} \int_{L_{z}} u_{, \alpha \beta} u_{, \alpha \beta} d x_{2}\right|_{\eta=t}+\left.\frac{1}{2} \int_{L_{z}} u_{, 1 \alpha} u_{, 1 \alpha} d x_{2}\right|_{\eta=t}
$$

gains its maximum value, i.e.,

$$
\begin{aligned}
m(z, \tilde{t}) & =\left.\frac{1}{2} \int_{L_{z}} u_{, \alpha \beta} u_{, \alpha \beta} d x_{2}\right|_{\eta=\tilde{t}}+\left.\frac{1}{2} \int_{L_{z}} u_{, 1 \alpha} u_{, 1 \alpha} d x_{2}\right|_{\eta=\tilde{t}} \\
& =\max _{0 \leq s \leq t}\left\{\left.\frac{1}{2} \int_{L_{z}} u_{, \alpha \beta} u_{, \alpha \beta} d x_{2}\right|_{\eta=s}+\left.\frac{1}{2} \int_{L_{z}} u_{, 1 \alpha} u_{, 1 \alpha} d x_{2}\right|_{\eta=s}\right\} .
\end{aligned}
$$

We now define

$$
t^{*}=\lim _{Z \rightarrow \infty}\left\{\sup _{0 \leq z \leq Z} \tilde{t}(z)\right\}
$$


SPATIAL BEHAVIOR OF SOLUTION FOR THE STOKES FLOW EQUATION 403

Following the same procedure as in producing $t^{*}$, we can also define $0 \leq$ $t_{1}^{*} \leq t, 0 \leq t_{2}^{*} \leq t$ such that

(3.3) $m_{1}\left(z, \tilde{t_{1}}\right)=\left.\frac{1}{2} \int_{L_{z}} u_{, \alpha \beta} u_{, \alpha \beta} d x_{2}\right|_{\eta=\tilde{t_{1}}}=\max _{0 \leq s \leq t}\left\{\left.\frac{1}{2} \int_{L_{z}} u_{, \alpha \beta} u_{, \alpha \beta} d x_{2}\right|_{\eta=s}\right\}$,

(3.4) $m_{2}\left(z, \tilde{t_{2}}\right)=\left.\frac{1}{2} \int_{L_{z}} u_{, 1 \alpha} u_{, 1 \alpha} d x_{2}\right|_{\eta=\tilde{t_{2}}}=\max _{0 \leq s \leq t}\left\{\left.\frac{1}{2} \int_{L_{z}} u_{, 1 \alpha} u_{, 1 \alpha} d x_{2}\right|_{\eta=s}\right\}$.

We also define

$$
t_{1}^{*}=\lim _{Z \rightarrow \infty}\left\{\sup _{0 \leq z \leq Z} \tilde{t_{1}}(z)\right\}
$$

and

$$
t_{2}^{*}=\lim _{Z \rightarrow \infty}\left\{\sup _{0 \leq z \leq Z} \tilde{t_{2}}(z)\right\} .
$$

From the definitions of $m_{1}\left(z, \tilde{t_{1}}\right), m_{2}\left(z, \tilde{t_{2}}\right) m(z, \tilde{t})$ and $t_{1}^{*}, t_{2}^{*}$, we can easily get

$$
m_{2}\left(z, t_{2}^{*}\right) \leq m_{1}\left(z, t_{1}^{*}\right) \leq m\left(z, t^{*}\right)
$$

Now, we write

$$
\begin{aligned}
\phi\left(z, t^{*}\right)= & -\int_{0}^{t^{*}} \int_{z_{0}}^{z} \int_{L_{\xi}} u_{, \alpha \eta} u_{, \alpha \eta} d A d \eta-\left.\frac{1}{2} \int_{z_{0}}^{z} \int_{L_{\xi}} u_{, \alpha \beta} u_{, \alpha \beta} d A\right|_{\eta=t^{*}} \\
& -\int_{0}^{t^{*}} \int_{z_{0}}^{z} \int_{L_{\xi}} u_{, 1 \alpha \beta} u_{, 1 \alpha \beta} d A d \eta-\left.\frac{1}{2} \int_{z_{0}}^{z} \int_{L_{\xi}} u_{, 1 \alpha} u_{, 1 \alpha} d A\right|_{\eta=t^{*}}+\phi\left(z_{0}, t^{*}\right) .
\end{aligned}
$$

From (2.21), we can get

$$
\begin{aligned}
\left|\phi\left(z, t^{*}\right)\right| \leq & \left|\int_{0}^{t^{*}} \int_{L_{z}}\left(u_{, \eta} u_{, 1 \eta}+u_{, \alpha \eta} u_{, 1 \alpha}-u_{, \eta} u_{, 1 \beta \beta}\right) d x_{2} d \eta\right| \\
& +\left|\int_{0}^{t^{*}} \int_{L_{z}}\left(u_{, \alpha \alpha} u_{, 1 \alpha \alpha}+u_{, 12} u_{, 2 \eta}\right) d x_{2} d \eta\right| .
\end{aligned}
$$

Making use of Schwarz's inequality, inequality (2.26), and Young's inequality, it yields

$$
\begin{aligned}
& \left|\int_{0}^{t^{*}} \int_{L_{z}} u_{, \eta} u_{, 1 \eta} d x_{2} d \eta\right| \\
\leq & h\left[\frac{\varepsilon_{1}}{2} \int_{0}^{t^{*}} \int_{L_{z}} u_{, 2 \eta} u_{, 2 \eta} d x_{2} d \eta+\frac{1}{2 \varepsilon_{1}} \int_{0}^{t^{*}} \int_{L_{z}} u_{, 1 \eta} u_{, 1 \eta} d x_{2} d \eta\right], \\
& \left|\int_{0}^{t^{*}} \int_{L_{z}} u_{, \alpha \eta} u_{, 1 \alpha} d x_{2} d \eta\right|
\end{aligned}
$$




$$
\begin{aligned}
& \leq \frac{\varepsilon_{2}}{2} \int_{0}^{t^{*}} \int_{L_{z}} u_{, \alpha \eta} u_{, \alpha \eta} d x_{2} d \eta+\frac{1}{2 \varepsilon_{2}} \int_{0}^{t^{*}} \int_{L_{z}} u_{, 1 \alpha} u_{, 1 \alpha} d x_{2} d \eta \\
& \leq \frac{\varepsilon_{2}}{2} \int_{0}^{t^{*}} \int_{L_{z}} u_{, \alpha \eta} u_{, \alpha \eta} d x_{2} d \eta+\left.\frac{1}{2 \varepsilon_{2}} t^{*} \int_{L_{z}} u_{, 1 \alpha} u_{, 1 \alpha} d x_{2}\right|_{\eta=t_{2}^{*}} \\
& \leq \frac{\varepsilon_{2}}{2} \int_{0}^{t^{*}} \int_{L_{z}} u_{, \alpha \eta} u_{, \alpha \eta} d x_{2} d \eta+\left.\frac{1}{2 \varepsilon_{2}} t^{*} \int_{L_{z}} u_{, \alpha \beta} u_{, \alpha \beta} d x_{2}\right|_{\eta=t_{1}^{*}}
\end{aligned}
$$

$$
\begin{aligned}
& \left|\int_{0}^{t^{*}} \int_{L_{z}} u_{, \eta} u_{, 1 \alpha \alpha} d x_{2} d \eta\right| \\
\leq & h\left[\frac{\varepsilon_{3}}{2} \int_{0}^{t^{*}} \int_{L_{z}} u_{, 2 \eta} u_{, 2 \eta} d x_{2} d \eta+\frac{1}{2 \varepsilon_{3}} \int_{0}^{t^{*}} \int_{L_{z}} u_{, 1 \alpha \alpha} u_{, 1 \beta \beta} d x_{2} d \eta\right] \\
\leq & h\left[\frac{\varepsilon_{3}}{2} \int_{0}^{t^{*}} \int_{L_{z}} u_{, 2 \eta} u_{, 2 \eta} d x_{2} d \eta+\frac{1}{2 \varepsilon_{3}} \int_{0}^{t^{*}} \int_{L_{z}} u_{, 1 \alpha \beta} u_{, 1 \alpha \beta} d x_{2} d \eta\right],
\end{aligned}
$$

$$
\begin{aligned}
& \left|\int_{0}^{t^{*}} \int_{L_{z}} u_{, \alpha \alpha} u_{, 1 \alpha \alpha} d x_{2} d \eta\right| \\
\leq & \frac{\varepsilon_{4}}{2} \int_{0}^{t^{*}} \int_{L_{z}} u_{, \alpha \alpha} u_{, \beta \beta} d x_{2} d \eta+\frac{1}{2 \varepsilon_{4}} \int_{0}^{t^{*}} \int_{L_{z}} u_{, \alpha \alpha 1} u_{, \alpha \alpha 1} d x_{2} d \eta \\
\leq & \left.\frac{\varepsilon_{4}}{2} t^{*} \int_{L_{z}} u_{, \alpha \alpha} u_{, \beta \beta} d x_{2}\right|_{\eta=t_{1}^{*}}+\frac{1}{2 \varepsilon_{4}} \int_{0}^{t^{*}} \int_{L_{z}} u_{, \alpha \alpha 1} u_{, \beta \beta 1} d x_{2} d \eta \\
\leq & \left.\frac{\varepsilon_{4}}{2} t^{*} \int_{L_{z}} u_{, \alpha \beta} u_{, \alpha \beta} d x_{2}\right|_{\eta=t_{1}^{*}}+\frac{1}{2 \varepsilon_{4}} \int_{0}^{t^{*}} \int_{L_{z}} u_{, 1 \alpha \beta} u_{, 1 \alpha \beta} d x_{2} d \eta,
\end{aligned}
$$

$$
\begin{aligned}
& \left|\int_{0}^{t^{*}} \int_{L_{z}} u_{, 12} u_{, 2 \eta} d x_{2} d \eta\right| \\
\leq & \frac{\varepsilon_{5}}{2} \int_{0}^{t^{*}} \int_{L_{z}} u_{, 12} u_{, 12} d x_{2} d \eta+\frac{1}{2 \varepsilon_{5}} \int_{0}^{t^{*}} \int_{L_{z}} u_{, 2 \eta} u_{, 2 \eta} d x_{2} d \eta \\
\leq & \left.\frac{\varepsilon_{5}}{2} t^{*} \int_{L_{z}} u_{, \alpha \beta} u_{, \alpha \beta} d x_{2}\right|_{\eta=t_{1}^{*}}+\frac{1}{2 \varepsilon_{5}} \int_{0}^{t^{*}} \int_{L_{z}} u_{, 2 \eta} u_{, 2 \eta} d x_{2} d \eta \\
\leq & \left.\frac{\varepsilon_{5}}{2} t^{*} \int_{L_{z}} u_{, \alpha \beta} u_{, \alpha \beta} d x_{2}\right|_{\eta=t_{1}^{*}}+\frac{1}{2 \varepsilon_{5}} \int_{0}^{t^{*}} \int_{L_{z}} u_{, 2 \eta} u_{, 2 \eta} d x_{2} d \eta .
\end{aligned}
$$

If we choose $\varepsilon_{1}=\frac{\sqrt{2}}{2}, \varepsilon_{2}=1, \varepsilon_{3}=\frac{\sqrt{2}}{2}, \varepsilon_{4}=1, \varepsilon_{5}=1$, by combining (3.5) and (3.7)-(3.12), we obtain

$$
\left|\phi\left(z, t^{*}\right)\right| \leq\left(\frac{3}{2} t^{*}+k\right)\left(-\frac{\partial \phi\left(z, t^{*}\right)}{\partial z}\right)
$$

where $k=\frac{\sqrt{2}}{2} h+1$. 
We may write (3.13) as two inequalities:

$$
-\frac{\partial \phi\left(z, t^{*}\right)}{\partial z} \geq \frac{-\phi\left(z, t^{*}\right)}{\frac{3}{2} t^{*}+k}
$$

and

$$
-\frac{\partial \phi\left(z, t^{*}\right)}{\partial z} \geq \frac{\phi\left(z, t^{*}\right)}{\frac{3}{2} t^{*}+k}
$$

The following discussion will be divided into two cases.

Case 1: If there exists a point $\left(z_{1}, t^{*}\right)$ such that $-\phi\left(z_{1}, t^{*}\right)>0$, then for all $z>z_{1},-\phi\left(z, t^{*}\right)>0$. An integration of (3.14) leads to

$$
-\phi\left(z, t^{*}\right) \geq-\phi\left(z_{1}, t^{*}\right) \exp \left(\frac{z-z_{1}}{\frac{3}{2} t^{*}+k}\right) .
$$

Case 2: If there doesn't exist a point $\left(z_{1}, t^{*}\right)$ such that $-\phi\left(z_{1}, t^{*}\right)>0$, then for all $z \geq 0, \phi\left(z, t^{*}\right) \geq 0$. We have, from (3.15),

$$
\phi\left(z, t^{*}\right) \leq \phi\left(0, t^{*}\right) \exp \left(\frac{-z}{\frac{3}{2} t^{*}+k}\right) .
$$

From $(3.16)$, we can get $\lim _{z \rightarrow \infty}\left(-\phi\left(z, t^{*}\right)\right)=\infty$, that is to say for $z$ large enough, we can get

$$
-\phi\left(z, t^{*}\right) \geq-2 \phi\left(z_{1}, t^{*}\right)
$$

From (3.6), we get

$$
\begin{aligned}
-\phi\left(z, t^{*}\right) \leq & 2 \int_{0}^{t^{*}} \int_{z_{1}}^{z} \int_{L_{\xi}} u_{, \alpha \eta} u_{, \alpha \eta} d A d \eta+\left.\int_{z_{1}}^{z} \int_{L_{\xi}} u_{, \alpha \beta} u_{, \alpha \beta} d A\right|_{\eta=t^{*}} \\
& +2 \int_{0}^{t^{*}} \int_{z_{1}}^{z} \int_{L_{\xi}} u_{, 1 \alpha \beta} u_{, 1 \alpha \beta} d A d \eta+\left.\int_{z_{1}}^{z} \int_{L_{\xi}} u_{, 1 \alpha} u_{, 1 \alpha} d A\right|_{\eta=t^{*}} \\
= & E_{1}\left(z, t^{*}\right) .
\end{aligned}
$$

Combining (3.16) and (3.18), we obtain

$$
\lim _{z \rightarrow \infty} \exp \left(\frac{z_{1}-z}{\frac{3}{2} t^{*}+k}\right) E_{1}\left(z, t^{*}\right) \geq-\phi\left(z, t^{*}\right) .
$$

Clearly, if $\lim _{z \rightarrow \infty} \phi\left(z, t^{*}\right)=0$, we can easily get from (3.6) that

$$
\begin{aligned}
\phi\left(z, t^{*}\right)= & \int_{0}^{t^{*}} \int_{z}^{\infty} \int_{L_{\xi}} u_{, \alpha \eta} u_{, \alpha \eta} d A d \eta+\left.\frac{1}{2} \int_{z}^{\infty} \int_{L_{\xi}} u_{, \alpha \beta} u_{, \alpha \beta} d A\right|_{\eta=t^{*}} \\
& +\int_{0}^{t^{*}} \int_{z}^{\infty} \int_{L_{\xi}} u_{, 1 \alpha \beta} u_{, 1 \alpha \beta} d A d \eta+\left.\frac{1}{2} \int_{z}^{\infty} \int_{L_{\xi}} u_{, 1 \alpha} u_{, 1 \alpha} d A\right|_{\eta=t^{*}}
\end{aligned}
$$


We have

$$
\begin{aligned}
E_{2}\left(z, t^{*}\right) & =\left.\frac{1}{2} \int_{z}^{\infty} \int_{L_{\xi}} u_{, \alpha \beta} u_{, \alpha \beta} d A\right|_{\eta=t^{*}}+\left.\frac{1}{2} \int_{z}^{\infty} \int_{L_{\xi}} u_{, 1 \alpha} u_{, 1 \alpha} d A\right|_{\eta=t^{*}} \\
& \leq \phi\left(z, t^{*}\right) .
\end{aligned}
$$

Summarizing all the results above, we conclude the following theorem:

Theorem 1. If $u$ is a solution of the initial boundary value problem (2.9)(2.14), then, either

$$
\lim _{z \longrightarrow \infty} \exp \left(\frac{z_{1}-z}{\frac{3}{2} t^{*}+k} E_{1}\left(z, t^{*}\right)\right) \geq-\phi\left(z_{1}, t^{*}\right),
$$

is satisfied, or the "energy" function $E_{2}(z, t)$ satisfies the estimates

$$
\begin{aligned}
E_{2}(z, t) \leq E_{2}\left(z, t^{*}\right) \leq \phi\left(z, t^{*}\right) & \leq \phi\left(0, t^{*}\right) \exp \left[\left(-\frac{1}{\frac{3}{2} t^{*}+k}\right) z\right] \\
& \leq \phi\left(0, t^{*}\right) \exp \left[\left(-\frac{1}{\frac{3}{2} t+k}\right) z\right],
\end{aligned}
$$

where $t^{*}$ is a value $($ see $(3.2))$ belonging to $(0, t]$.

\section{Upper bound for the total energy}

We now show how a explicit bound can be determined for $\phi\left(0, t^{*}\right)$ which occurred in (3.23).

Under the case of decay, we define

$$
\begin{aligned}
F\left(z, t^{*}\right)= & \phi\left(z, t^{*}\right) \\
= & \int_{0}^{t^{*}} \int_{z}^{\infty} \int_{L_{\xi}} u_{, \alpha \eta} u_{, \alpha \eta} d A d \eta+\left.\frac{1}{2} \int_{z}^{\infty} \int_{L_{\xi}} u_{, \alpha \beta} u_{, \alpha \beta} d A\right|_{\eta=t^{*}} \\
& +\int_{0}^{t^{*}} \int_{z}^{\infty} \int_{L_{\xi}} u_{, 1 \alpha \beta} u_{, 1 \alpha \beta} d A d \eta+\left.\frac{1}{2} \int_{z}^{\infty} \int_{L_{\xi}} u_{, 1 \alpha} u_{, 1 \alpha} d A\right|_{\eta=t^{*}}
\end{aligned}
$$

If we define

$$
F\left(z, t^{*}\right)=F_{1}\left(z, t^{*}\right)+F_{2}\left(z, t^{*}\right),
$$

where

$$
\begin{aligned}
& F_{1}\left(z, t^{*}\right)=\int_{0}^{t^{*}} \int_{z}^{\infty} \int_{L_{\xi}} u_{, \alpha \eta} u_{, \alpha \eta} d A d \eta+\left.\frac{1}{2} \int_{z}^{\infty} \int_{L_{\xi}} u_{, \alpha \beta} u_{, \alpha \beta} d A\right|_{\eta=t^{*}}, \\
& F_{2}\left(z, t^{*}\right)=\int_{0}^{t^{*}} \int_{z}^{\infty} \int_{L_{\xi}} u_{, 1 \alpha \beta} u_{, 1 \alpha \beta} d A d \eta+\left.\frac{1}{2} \int_{z}^{\infty} \int_{L_{\xi}} u_{, 1 \alpha} u_{, 1 \alpha} d A\right|_{\eta=t^{*}},
\end{aligned}
$$

and

(4.3) $\quad F_{u}\left(z, t^{*}\right)=F_{1}\left(z, t^{*}\right)$, 
SPATIAL BEHAVIOR OF SOLUTION FOR THE STOKES FLOW EQUATION 407

$$
\begin{aligned}
& \text { (4.4) } F_{w}\left(z, t^{*}\right)=\int_{0}^{t^{*}} \int_{z}^{\infty} \int_{L_{\xi}} w_{, \alpha \eta} w_{, \alpha \eta} d A d \eta+\left.\frac{1}{2} \int_{z}^{\infty} \int_{L_{\xi}} w_{, \alpha \beta} w_{, \alpha \beta} d A\right|_{\eta=t^{*}}, \\
& \text { (4.5) } F_{u w}\left(z, t^{*}\right)=\int_{0}^{t^{*}} \int_{z}^{\infty} \int_{L_{\xi}} u_{, \alpha \eta} w_{, \alpha \eta} d A d \eta+\left.\frac{1}{2} \int_{z}^{\infty} \int_{L_{\xi}} u_{, \alpha \beta} w_{, \alpha \beta} d A\right|_{\eta=t^{*}},
\end{aligned}
$$

where $w\left(x_{1}, x_{2}, t\right)$ is an arbitrary smooth function defined on $\Omega_{0}$ that satisfies the same boundary conditions as $u$.

By using the Schwarz's inequality, we get

$$
\sqrt{F_{u}\left(z, t^{*}\right) F_{w}\left(z, t^{*}\right)} \geq F_{u w}\left(z, t^{*}\right) .
$$

It is clear that

$$
\begin{aligned}
F_{u w}\left(0, t^{*}\right)= & F_{u}\left(0, t^{*}\right)+\int_{0}^{t^{*}} \int_{0}^{\infty} \int_{L_{\xi}} u_{, \alpha \eta}\left(w_{, \alpha \eta}-u_{, \alpha \eta}\right) d A d \eta \\
& +\left.\frac{1}{2} \int_{0}^{\infty} \int_{L_{\xi}} u_{, \alpha \beta}\left(w_{, \alpha \beta}-u_{, \alpha \beta}\right) d A\right|_{\eta=t^{*}} \\
= & F_{u}\left(0, t^{*}\right)+I_{1}+I_{2} .
\end{aligned}
$$

Making use of the divergence theorem, and (2.9)-(2.14), we obtain

$$
\begin{aligned}
I_{1}= & -\int_{0}^{t^{*}} \int_{0}^{\infty} \int_{L_{\xi}} u_{, \alpha \alpha \eta}\left(w_{, \eta}-u_{, \eta}\right) d A d \eta \\
= & \int_{0}^{t^{*}} \int_{0}^{\infty} \int_{L_{\xi}} u_{, \alpha \beta \beta}\left(w_{, \alpha \eta}-u_{, \alpha \eta}\right) d A d \eta \\
= & -\int_{0}^{t^{*}} \int_{0}^{\infty} \int_{L_{\xi}} u_{, \alpha \beta}\left(w_{, \alpha \beta \eta}-u_{, \alpha \beta \eta}\right) d A d \eta \\
\geq & \left.\frac{1}{2} \int_{0}^{\infty} \int_{L_{\xi}} u_{, \alpha \beta} u_{, \alpha \beta} d A\right|_{\eta=t^{*}}-\left.\frac{\varepsilon_{1}}{2} \int_{0}^{\infty} \int_{L_{\xi}} u_{, \alpha \beta} u_{, \alpha \beta} d A\right|_{\eta=t^{*}} \\
& -\frac{1}{2 \varepsilon_{1}} t^{*} \int_{0}^{t^{*}} \int_{0}^{\infty} \int_{L_{\xi}} w_{, \alpha \beta \eta} w_{, \alpha \beta \eta} d A d \eta,
\end{aligned}
$$

$$
\begin{aligned}
I_{2}= & \left.\frac{1}{2} \int_{0}^{\infty} \int_{L_{\xi}} u_{, \alpha \beta} w_{, \alpha \beta} d A\right|_{\eta=t^{*}}-\left.\frac{1}{2} \int_{0}^{\infty} \int_{L_{\xi}} u_{, \alpha \beta} u_{, \alpha \beta} d A\right|_{\eta=t^{*}} \\
\geq & -\left.\frac{\varepsilon_{2}}{4} \int_{0}^{\infty} \int_{L_{\xi}} u_{, \alpha \beta} u_{, \alpha \beta} d A\right|_{\eta=t^{*}}-\left.\frac{1}{4 \varepsilon_{2}} \int_{0}^{\infty} \int_{L_{\xi}} w_{, \alpha \beta} w_{, \alpha \beta} d A\right|_{\eta=t^{*}} \\
& -\left.\frac{1}{2} \int_{0}^{\infty} \int_{L_{\xi}} u_{, \alpha \beta} u_{, \alpha \beta} d A\right|_{\eta=t^{*}} .
\end{aligned}
$$

Setting $\varepsilon_{1}=1, \varepsilon_{2}=\frac{1}{2}$, then combining (4.6)-(4.8) and (4.9), we are led to

$$
\sqrt{F_{u}\left(0, t^{*}\right) F_{w}\left(0, t^{*}\right)} \geq \frac{3}{4} F_{u}\left(0, t^{*}\right)-Q\left(w\left(0, t^{*}\right)\right),
$$


where we have

$Q\left(w\left(0, t^{*}\right)\right)=\frac{t^{*}}{2} \int_{0}^{t^{*}} \int_{0}^{\infty} \int_{L_{\xi}} w_{, \alpha \beta \eta} w_{, \alpha \beta \eta} d A d \eta+\left.\frac{1}{2} \int_{0}^{\infty} \int_{L_{\xi}} w_{, \alpha \beta} w_{, \alpha \beta} d A\right|_{\eta=t^{*}}$.

From (4.10), we can conclude

$$
\frac{3}{4}\left(\sqrt{F_{u}\left(0, t^{*}\right)}-\frac{2}{3}\left(\sqrt{F_{w}\left(0, t^{*}\right)}\right)^{2} \leq \frac{1}{3}\left(F_{w}\left(0, t^{*}\right)+3 Q\left(w\left(0, t^{*}\right)\right)\right),\right.
$$

which implies that

$$
\sqrt{F_{u}\left(0, t^{*}\right)} \leq \frac{2}{3} \sqrt{F_{w}\left(0, t^{*}\right)}+\frac{2}{3}\left(\sqrt{F_{w}\left(0, t^{*}\right)}+3 Q\left(w\left(0, t^{*}\right)\right)\right)^{\frac{1}{2}} .
$$

Squaring and making use of the arithmetic-geometric mean inequality, we obtain

$$
F_{u}\left(0, t^{*}\right) \leq \frac{16}{9} F_{w}\left(0, t^{*}\right)+\frac{8}{3} Q\left(w\left(0, t^{*}\right)\right) .
$$

Since we don't know the value of $t^{*}$, the bound for $F_{u}\left(0, t^{*}\right)$ can be made explicit only if we can eliminate the dependent on $t^{*}$. Clearly, we know, for any function $\varphi$ such that $\varphi(x, 0)=0$,

$$
\left.\int_{L_{z}} \varphi^{2} d x_{2}\right|_{\eta=t^{*}}=2 \int_{0}^{t^{*}} \int_{L_{z}} \varphi \varphi, \eta d x_{2} d \eta \leq \int_{0}^{t} \int_{L_{z}} \varphi^{2} d x_{2} d \eta+\int_{0}^{t} \int_{L_{z}} \varphi_{, \eta}^{2} d x_{2} d \eta .
$$

Using (4.14), we obtain

$$
\begin{aligned}
F_{w}\left(0, t^{*}\right)= & \int_{0}^{t^{*}} \int_{0}^{\infty} \int_{L_{\xi}} w_{, \alpha \eta} w_{, \alpha \eta} d A d \eta+\left.\frac{1}{2} \int_{0}^{\infty} \int_{L_{\xi}} w_{, \alpha \beta} w_{, \alpha \beta} d A\right|_{\eta=t^{*}} \\
\leq & \int_{0}^{t} \int_{0}^{\infty} \int_{L_{\xi}} w_{, \alpha \eta} w_{, \alpha \eta} d A d \eta+\frac{1}{2} \int_{0}^{t} \int_{0}^{\infty} \int_{L_{\xi}} w_{, \alpha \beta} w_{, \alpha \beta} d A d \eta \\
& +\frac{1}{2} \int_{0}^{t} \int_{0}^{\infty} \int_{L_{\xi}} w_{, \alpha \beta \eta} w_{, \alpha \beta \eta} d A d \eta \\
= & \bar{F}_{w}(0, t),
\end{aligned}
$$

and

$$
\begin{aligned}
Q\left(w\left(0, t^{*}\right)\right)= & \frac{t^{*}}{2} \int_{0}^{t^{*}} \int_{0}^{\infty} \int_{L_{\xi}} w_{, \alpha \beta \eta} w_{, \alpha \beta \eta} d A d \eta+\left.\frac{1}{2} \int_{0}^{\infty} \int_{L_{\xi}} w_{, \alpha \beta} w_{, \alpha \beta} d A\right|_{\eta=t^{*}} \\
\leq & \frac{t}{2} \int_{0}^{t} \int_{0}^{\infty} \int_{L_{\xi}} w_{, \alpha \beta \eta} w_{, \alpha \beta \eta} d A d \eta+\frac{1}{2} \int_{0}^{t} \int_{0}^{\infty} \int_{L_{\xi}} w_{, \alpha \beta} w_{, \alpha \beta} d A d \eta \\
& +\frac{1}{2} \int_{0}^{t} \int_{0}^{\infty} \int_{L_{\xi}} w_{, \alpha \beta \eta} w_{, \alpha \beta \eta} d A d \eta \\
= & \bar{Q}(w(0, t)) .
\end{aligned}
$$


We can get

$$
F_{u}\left(0, t^{*}\right) \leq \frac{16}{9} \bar{F}_{w}(0, t)+\frac{8}{3} \bar{Q}(w(0, t)) .
$$

We now define $w\left(x_{1}, x_{2}, t\right)$ as

$$
w\left(x_{1}, x_{2}, t\right)=\left[\int_{0}^{x_{2}} \frac{\partial g_{1}}{\partial \xi}(\xi, t) d \xi+x_{1}\left(g_{2}\left(x_{2}, t\right)+\int_{0}^{x_{2}} \frac{\partial g_{1}}{\partial \xi}(\xi, t) d \xi\right)\right] e^{-x_{1}},
$$

Obviously, it is readily to verify $w\left(x_{1}, x_{2}, t\right)$ satisfying the same initial-boundary value conditions as $u\left(x_{1}, x_{2}, t\right)$.

Combining (4.15) and (4.16), we can say we have bounded $F_{u}\left(0, t^{*}\right)$.

The next step is to bound $F_{2}\left(0, t^{*}\right)$.

We will use the following lemma:

Lemma 1. If $\phi\left(z, t^{*}\right)$ decays to zero as $z \rightarrow \infty$, then for arbitrary $z \geq 0$, $z_{0}>0$,

$$
z_{0}^{2} F_{2}\left(z+z_{0}, t^{*}\right) \leq 8 t F_{1}\left(z, t^{*}\right)
$$

Proof. We adapt the notation

$$
\begin{aligned}
\tilde{F}_{2}\left(z, t^{*}\right)= & \int_{0}^{t^{*}} \int_{z}^{\infty} \int_{L_{\xi}}(\xi-z)^{2} u_{, 1 \alpha \beta} u_{, 1 \alpha \beta} d A d \eta \\
& +\left.\frac{1}{2} \int_{z}^{\infty} \int_{L_{\xi}}(\xi-z)^{2} u_{, 1 \alpha} u_{, 1 \alpha} d A\right|_{\eta=t^{*}}
\end{aligned}
$$

Obviously, we have the following identity

$$
0=\int_{0}^{t^{*}} \int_{z}^{\infty} \int_{L_{\xi}}(\xi-z)^{2} u_{, 11}\left(\Delta^{2} u-\Delta u_{, \eta}\right) d A d \eta
$$

By applying the divergence theorem repeatedly and using the initial-boundary value conditions, we obtain

$$
\begin{aligned}
& 0=-\int_{0}^{t^{*}} \int_{z}^{\infty} \int_{L_{\xi}}(\xi-z)^{2} u_{, 1 \alpha \beta} u_{, 1 \alpha \beta} d A d \eta+\int_{0}^{t^{*}} \int_{z}^{\infty} \int_{L_{\xi}} u_{, \alpha \beta} u_{, \alpha \beta} d A d \eta \\
&-2 \int_{0}^{t^{*}} \int_{z}^{\infty} \int_{L_{\xi}} u_{, 12} u_{, 12} d A d \eta-\left.\frac{1}{2} \int_{z}^{\infty} \int_{L_{\xi}}(\xi-z)^{2} u_{, 1 \alpha} u_{, 1 \alpha} d A\right|_{\eta=t^{*}} \\
&(4.20) \quad+2 \int_{0}^{t^{*}} \int_{z}^{\infty} \int_{L_{\xi}}(\xi-z) u_{, 12} u_{, 2 \eta} d A d \eta .
\end{aligned}
$$

Inserting (4.20) into (4.18), we find

$$
\tilde{F}_{2}\left(z, t^{*}\right) \leq \int_{0}^{t^{*}} \int_{z}^{\infty} \int_{L_{\xi}} u_{, \alpha \beta} u_{, \alpha \beta} d A d \eta+2\left|\int_{0}^{t^{*}} \int_{z}^{\infty} \int_{L_{\xi}}(\xi-z) u_{, 12} u_{, 2 \eta} d A d \eta\right| .
$$


We have

$$
\begin{aligned}
& \int_{0}^{t^{*}} \int_{z}^{\infty} \int_{L_{\xi}} u_{, \alpha \beta} u_{, \alpha \beta} d A d \eta \leq\left. t^{*} \int_{z}^{\infty} \int_{L_{\xi}} u_{, \alpha \beta} u_{, \alpha \beta} d A\right|_{\eta=t_{1}^{*}} \\
\leq & t\left(\left.\int_{z}^{\infty} \int_{L_{\xi}} u_{, \alpha \beta} u_{, \alpha \beta} d A\right|_{\eta=t^{*}}+\left.\int_{z}^{\infty} \int_{L_{\xi}} u_{, 1 \alpha} u_{, 1 \alpha} d A\right|_{\eta=t^{*}}\right) \\
\leq & 4 t F_{1}\left(z, t^{*}\right) .
\end{aligned}
$$

Using the Schwarz's inequality, we have

$$
\begin{aligned}
2\left|\int_{0}^{t^{*}} \int_{z}^{\infty} \int_{L_{\xi}}(\xi-z) u_{, 12} u_{, 2 \eta} d A d \eta\right| \leq & \left.\varepsilon \int_{z}^{\infty} \int_{L_{\xi}}(\xi-z)^{2} u_{, 1 \alpha} u_{, 1 \alpha} d A\right|_{\eta=t^{*}} \\
& +\frac{1}{\varepsilon} t^{*} \int_{0}^{t^{*}} \int_{z}^{\infty} \int_{L_{\xi}} u_{, 2 \eta}^{2} d A d \eta .
\end{aligned}
$$

If we choose $\varepsilon=\frac{1}{4}$, combining (4.22) and (4.23), we obtain

$$
\tilde{F}_{2}\left(z, t^{*}\right) \leq 8 t F_{1}\left(z, t^{*}\right) .
$$

We note that for arbitrary $z_{0}>0$,

$$
\tilde{F}_{2}\left(z, t^{*}\right) \geq \int_{0}^{t^{*}} \int_{z+z_{0}}^{\infty} \int_{L_{\xi}}(\xi-z)^{2} u_{, 1 \alpha \beta} u_{, 1 \alpha \beta} d A d \eta \geq z_{0}^{2} F_{2}\left(z+z_{0}, t^{*}\right) .
$$

In view of (4.25), we have proved Lemma 1.

For we know,

$$
\left.\int_{z}^{\infty} \int_{L_{\xi}} u_{, 1 \alpha} u_{, 1 \alpha} d A\right|_{\eta=t^{*}} \leq\left.\int_{z}^{\infty} \int_{L_{\xi}} u_{, \alpha \beta} u_{, \alpha \beta} d A\right|_{\eta=t^{*}}
$$

Thus, we only need to bound $F_{3}\left(0, t^{*}\right)=\int_{0}^{t^{*}} \int_{z}^{\infty} \int_{L_{\xi}} u_{, 1 \alpha \beta} u, 1 \alpha \beta d A d \eta$.

Now, we begin to bound

$$
\begin{aligned}
\int_{0}^{t^{*}} \int_{0}^{\infty} \int_{L_{\xi}} u_{, 1 \alpha \beta} u_{, 1 \alpha \beta} d A d \eta= & \int_{0}^{t^{*}} \int_{0}^{1} \int_{L_{\xi}} u_{, 1 \alpha \beta} u_{, 1 \alpha \beta} d A d \eta \\
& +\int_{0}^{t^{*}} \int_{1}^{\infty} \int_{L_{\xi}} u_{, 1 \alpha \beta} u_{, 1 \alpha \beta} d A d \eta
\end{aligned}
$$

Setting $z=0, z_{0}=1$ in (4.17) leads to

$$
\int_{0}^{t^{*}} \int_{1}^{\infty} \int_{L_{\xi}} u_{, 1 \alpha \beta} u_{, 1 \alpha \beta} d A d \eta \leq 8 t F_{1}\left(0, t^{*}\right) .
$$

Using (4.24), and setting $z=0$, we obtain

$$
\int_{0}^{t^{*}} \int_{0}^{\infty} \int_{L_{\xi}} \xi^{2} u_{, 1 \alpha \beta} u_{, 1 \alpha \beta} d A d \eta \leq 8 t F_{1}\left(0, t^{*}\right) .
$$


Then (4.29) is equivalent to

$$
\int_{0}^{t^{*}} \int_{0}^{\infty} \int_{L_{\xi}} \psi_{, \xi} \psi_{, \xi} d A d \eta \leq 8 t F_{1}\left(0, t^{*}\right),
$$

where $\psi=u_{, \alpha \beta}$.

For the first integral on the right hand side of (4.25), making a variat transformation $x_{1}=\frac{1}{\xi}$, and using the inequality (4.30), we deduce that

$$
\begin{aligned}
\int_{0}^{t^{*}} \int_{0}^{1} \int_{L_{\xi}} u_{, 1 \alpha \beta} u_{, 1 \alpha \beta} d A d \eta & =\int_{0}^{t^{*}} \int_{1}^{\infty} \int_{L_{\xi}} \xi^{2} u_{, \xi \alpha \beta} u_{, \xi \alpha \beta} d A d \eta \\
& \leq \int_{0}^{t^{*}} \int_{0}^{\infty} \int_{L_{\xi}} \xi^{2} u_{, \xi \alpha \beta} u_{, \xi \alpha \beta} d A d \eta \leq 8 t F_{1}\left(0, t^{*}\right) .
\end{aligned}
$$

Combining (4.28) and (4.31), we finally obtain

$$
\int_{0}^{t^{*}} \int_{0}^{\infty} \int_{L_{\xi}} u_{, 1 \alpha \beta} u_{, 1 \alpha \beta} d A d \eta \leq 16 t F_{1}\left(0, t^{*}\right) .
$$

In view of (4.15), (4.26) and (4.32), we can conclude that we have bounded $F_{2}\left(0, t^{*}\right)$.

Thus, we have bounded $\phi\left(0, t^{*}\right)$ in terms of data.

\section{References}

[1] J. N. Flavin, On Knowles'version of Saint-Venant's principle in two-dimensional elastostatics, Arch. Rational Mech. Anal. 53 (1973/74), 366-375.

[2] J. N. Flavin, R. J. Knops, and L. E. Payne, Asymptotic behaviour of solutions to semilinear elliptic equations on the half-cylinder, Z. Angew. Math. Phys. 43 (1992), no. 3, 405-421.

[3] C. O. Horgan, Recent developments concerning Saint-Venant's principle: an update, AMR 42 (1989), no. 11, part 1, 295-303.

[4] _ Recent development concerning Saint-Venant's principle: a second update, Applied Mechanics Reviews 49 (1996), 101-111.

[5] Decay estimates for the biharmonic equation with applications to Saint-Venant principles in plane elasticity and Stokes flows, Quart. Appl. Math. 47 (1989), no. 1, $147-157$.

[6] C. O. Horgan and J. K. Knowles, Recent developments concerning Saint-Venant's principle, Adv. in Appl. Mech. 23 (1983), 179-269.

[7] C. O. Horgan and L. E. Payne, Phragmén-Lindelöf type results for harmonic functions with nonlinear boundary conditions, Arch. Rational Mech. Anal. 122 (1993), no. 2, $123-144$.

[8] J. K. Knowles, On the spatial decay of solutions of the heat equation, Z. Angew. Math. Phys. 22 (1971), 1050-1056.

[9] _ An energy estimate for the biharmonic equation and its application to SaintVenant's principle in plane elastostatics, Indian J. Pure Appl. Math. 14 (1983), no. 7, 791-805.

[10] C. Lin, Spatial decay estimates and energy bounds for the Stokes flow equation, Stability and Appl. Anal. of Continuous Media 2 (1992), 249-264.

[11] C. Lin and L. E. Payne, Phragmén-Lindelöf type results for second order quasilinear parabolic equations in $R^{2}$, Z. Angew. Math. Phys. 45 (1994), no. 2, 294-311. 
[12] L. E. Payne and P. W. Schaefer, Some Phragmén-Lindelöf type results for the biharmonic equation, Z. Angew. Math. Phys. 45 (1994), no. 3, 414-432.

[13] J. C. Song, Improved decay estimates in time-dependent Stokes flow, J. Math. Anal. Appl. 288 (2003), no. 2, 505-517.

YAN LIU

Department of Applied Mathematics

Guangdong University of Finance

Guangzhou 510521, P. R. China

E-mail address: liuyan99021324@tom.com

WENHUi LiaO

Department of ApPlied Mathematics

Guangdong University of Finance

Guangzhou 510521, P. R. China

E-mail address: liaowenhui526@sina.com

Changhao Lin

School of Mathematical Sciences

South China Normal University

Guangzhou 510631, P. R. China

E-mail address: linchh@scnu.edu.cn 